\title{
A novel differential-based protection scheme for intertie zone of large-scale centralized DFIG wind farms
}

\begin{abstract}
Doubly Fed Induction Generator (DFIG) wind farms as a reliable source of renewable energy have been increasingly integrated to power grid in the last two decades. Distance protection relay has continuously been the most common protection scheme implemented for wind farm intertie zone, however, nowadays with the enormous penetration of large-scale DFIG wind farms, these relays are no longer reliable, due to their incapability of providing accurate impedance measurement during internal and external faults, thus, causing maloperation, false tripping or delayed operation. In this study, a differential-based protective relay scheme is developed in Matlab/Simulink in order to provide reliable protection for wind farm intertie zone. Also, an aggregated model of a large-scale centralized wind farm has been designed to examine the performance of the proposed protection technique by imposing numerous internal and external faults at different locations. The results proved that differential-based protection relays (DBPR) are able to provide reliable, efficient and robust protection for the intertie zone of wind farms. Because, the differential relays provide high sensitivity, swift operation, immunity to power swings, and also inherently being a unit protection-based scheme that is extremely advantageous compared to distance relays. Moreover, unlike distance relays DBPRs do not require to cope with "underreach" and "overreach" characteristics, resulting in no false tripping during external faults.
\end{abstract}

Keyword: Distance relay; Differential relay; Overcurrent relay; Wind farm protection; Power system protection; Intertie system; Protective relays coordination 